\title{
Ultrasound assisted in-situ separation of sophorolipids in multi-phase fermentation system to achieve efficient production of Candida bombicola
}

\author{
Yang Chen ${ }^{1}$, Xu Tang ${ }^{1}$, Ya Li ${ }^{1}$, Chang Liu ${ }^{1}$, Yingping Zhuang ${ }^{2}$, Xiwei Tian ${ }^{3}$, and Ju Chu ${ }^{1}$ \\ ${ }^{1}$ East China University of Science and Technology State Key Laboratory of Bioreactor \\ Engineering \\ ${ }^{2}$ East China University of Science \& Technology \\ ${ }^{3}$ State KeyLaboratory of Bioreactor Engineering,
}

September 25, 2021

\begin{abstract}
Sophorolipids (SLs) are regarded as one of the most promising biosurfactants. However, high production costs are the main obstacle to extended SLs application. Semi-continuous fermentation, which is based on in-situ separation, is a promising technology for achieving high SLs productivity. In this study, the sedimentation mechanism of SLs was analyzed. The formation of a hydrophobic mixture of SLs and rapeseed oil was a key factor in sedimentation. And the hydrophobicity and density of the mixture determined SLs sedimentation rate. On this basis, ultrasonic enhanced sedimentation technology (UEST) was introduced, by which the sedimentation rates were increased by $46.9 \%$ to $485.4 \%$ with different ratio of rapeseed oil to SLs. UEST-assisted real-time in-situ separation and semi-continuous fermentation were performed. SLs productivity and yield were $2.15 \mathrm{~g} / \mathrm{L} / \mathrm{h}$ and $0.58 \mathrm{~g} / \mathrm{g}$, respectively, simultaneously the loss ratio of cells, glucose, and rapeseed oil were significantly reduced. This study provides the new horizon for optimization of the SLs fermentation process.
\end{abstract}

Ultrasound assisted in-situseparation of sophorolipids in multi-phase fermentation system to achieve efficient production of Candida bombicola

Yang Chen ${ }^{1}$, Xu Tang ${ }^{1}$, Ya Li ${ }^{1}$, Chang Liu ${ }^{1}$, Yingping Zhuang ${ }^{1}$, Xiwei Tian ${ }^{1 *}$, Ju Chu ${ }^{1 *}$

${ }^{1}$ State Key Laboratory of Bioreactor Engineering, East China University of Science and Technology, Shanghai 200237, China

*Author for correspondence

Address:

Ju Chu Xiwei Tian

State Key Laboratory of Bioreactor Engineering, East China University of Science and Technology

P.O. box 329, 130 Meilong Road, Shanghai 200237, People's Republic of China

Tel: +86-21-64253021; Tel: +86-21-64253853

E-mail address: juchu@ecust.edu.cn (Ju Chu), tahfy@163.com (Xiwei Tian)

Keywords: Sophorolipids; multiphase system; ultrasonic enhanced sedimentation; real-time; in-situ separation 
Abbreviations: Sophorolipids: SLs; ultrasonic enhanced sedimentation technology: UEST; The ratio of rapeseed oil to SLs: O/S;

\begin{abstract}
Sophorolipids (SLs) are regarded as one of the most promising biosurfactants. However, high production costs are the main obstacle to extended SLs application. Semi-continuous fermentation, which is based on in-situ separation, is a promising technology for achieving high SLs productivity. In this study, the sedimentation mechanism of SLs was analyzed. The formation of a hydrophobic mixture of SLs and rapeseed oil was a key factor in sedimentation. And the hydrophobicity and density of the mixture determined SLs sedimentation rate. On this basis, ultrasonic enhanced sedimentation technology (UEST) was introduced, by which the sedimentation rates were increased by $46.9 \%$ to $485.4 \%$ with different ratio of rapeseed oil to SLs. UESTassisted real-timein-situ separation and semi-continuous fermentation were performed. SLs productivity and yield were $2.15 \mathrm{~g} / \mathrm{L} / \mathrm{h}$ and $0.58 \mathrm{~g} / \mathrm{g}$, respectively, simultaneously the loss ratio of cells, glucose, and rapeseed oil were significantly reduced. This study provides the new horizon for optimization of the SLs fermentation process.
\end{abstract}

\title{
1. Introduction
}

Sophorolipids (SLs), which are regarded as one of the most promising biosurfactants, have been widely used in cosmetic, food, pharmaceutical fields due to their low toxicity and high biodegradability. ${ }^{[1,2]}$. Many microorganisms can synthesize SLs, including Candida, Pichia, andRhodotorula.$^{[3-5]}$ Candida bombicola is the most widely adopted specie due to its high efficiency of SLs production. ${ }^{[6]}$ SLs are the mixtures of many structural derivatives consisting of two parts, a hydrophilic sophorose and a hydrophobic hydroxyl fatty acid with general 16-18 carbon atoms. ${ }^{[7]}$ The differences of SLs structures are dependent on the degree of acetylation, the length of carbon chain, the unsaturation of fatty acids, and internal esterification, which further determined the properties of SLs. ${ }^{[8-10]}$ Therefore, SLs fermentation process is a complex multiphase system of gas, liquid, and solid, not only containing SLs with different structural properties (solid crystallization, oily, etc.), but also possessing bubbles, lipids, glucose, cells, and other substances in the fermentation broth. This poses a great challenge to the effective regulation and optimization of the fermentation process.

Biosurfactants have the advantages of green production and environmental friendliness, compared with chemical surfactants. However, the high production cost of SLs is still the main factor that hinders their development and application. ${ }^{[1-12]}$ Therefore, it is necessary to develop adequate process control strategies to improve production efficiency. To date, great progress has been made on SLs optimization in batch fermentations. However, the raise of SLs concentration during fermentation process leads to a significant increase in viscosity, which further results in the difficulty in mixing and mass transfer, and finally causing a sharp decline in fermentation efficiency. ${ }^{[13]}$ Semi-continuous fermentation basing onin-situ separation of products can segregate part of SLs from the fermentation broth, thereby improving the rheological characteristics of broth and maintaining efficient synthesis of SLs. Different types of devices have been developed to improve the efficiency of in-situseparation. ${ }^{[14]}$ In this regard, gravity sedimentation is the main method for in-situ separation of SLs. Zhang found that the density of the fermentation broth could be adjusted by controlling the glucose concentration, so as to achieve the sedimentation of SLs. ${ }^{[15]}$ Subsequently, genetically modified soybean oil was used to achieve the sedimentation and separation of SLs. Although these studies have achieved good results, the mechanism of SLs sedimentation in a heterogeneous fermentation system has not yet been clearly elucidated, thus it is difficult to rationally control SLs sedimentation and separation.

Generally, a large number of cells is lost during SLs in-situ separation process, so the efficiency of subsequent SLs synthesis will be significantly affected. Moreover, SLs have a strong affinity with oil, which also leads to the loss of oil, thereby reducing the substrate conversion rate. On the other hand, the enhancement of separation efficiency also contributes to the efficient production of SLs. Ultrasonic enhanced sedimentation technology (UEST) consists of the use of particle agglomeration by acoustophoresis, which causes increased gravitational force from larger effective particle size to increase the sedimentation rate. UEST has been applied in improving microalgae production, sludge settling and dehydration efficiency. ${ }^{[16,17]}$ Otherwise, 
Palme reported that UEST was applied in SLs fermentation forin-situ separation of cells, and the cell recovery was up to $99.0 \%$ without affecting the cell activity. However, it should be noted that only $10 \%$ of SLs could be recovered by this strategy. ${ }^{[18]}$

In this study, the influences of rapeseed oil concentrations on SLs morphology and sedimentation were investigated, and then the mechanism of SLs sedimentation was further discussed. On this basis, UEST was introduced for in-situ separation of SLs, and finally a rational semi-continuous fermentation process was developed for highly efficient SLs production.

\section{Material and methods}

2.1. Microorganism, media, and culture conditions

C. bombicola ATCC 22214 was obtained from the Guangdong Culture Collection Center (China) and stored at -80 in $20 \%$ glycerol solution. The seed medium consisted of $50 \mathrm{~g} / \mathrm{L}$ glucose, $1 \mathrm{~g} / \mathrm{L} \mathrm{KH}_{2} \mathrm{PO}_{4}, 4 \mathrm{~g} / \mathrm{L}$ $\left(\mathrm{NH}_{4}\right)_{2} \mathrm{SO}_{4}, 0.5 \mathrm{~g} / \mathrm{L} \mathrm{MgSO}{ }_{4} * 7 \mathrm{H}_{2} \mathrm{O}$, and $10 \mathrm{~g} / \mathrm{L}$ corn steep liquor. The seed was cultured in a $1 \mathrm{~L}$ baffled shake flask with $200 \mathrm{~mL}$ working volume at $200 \mathrm{rpm}$ and 25 for $48 \mathrm{~h}$.

The initial fermentation medium in a $5 \mathrm{~L}$ bioreactor (Shanghai Guoqiang Bioengineering Equipment Co., Ltd., China) consisted of glucose $100 \mathrm{~g} / \mathrm{L}, \mathrm{KH}_{2} \mathrm{PO}_{4} 1 \mathrm{~g} / \mathrm{L},\left(\mathrm{NH}_{4}\right)_{2} \mathrm{SO}_{4} 4 \mathrm{~g} / \mathrm{L}, \mathrm{MgSO}_{4} * 7 \mathrm{H}_{2} \mathrm{O} 0.5 \mathrm{~g} / \mathrm{L}$, corn steep liquor $10 \mathrm{~g} / \mathrm{L}$. All culture media were sterilized at 115 for $30 \mathrm{~min}$. The initial volume of $2.5 \mathrm{~L}$ with an inoculum of $3 \%$ was cultured at 25 for $168 \mathrm{~h}$. Aeration at $0.5 \mathrm{vvm}$ and dissolved oxygen above $30 \%$ of the saturation concentration were maintained by adjusting the agitation in a stepwise manner. A pH of 3.5 was maintained by addition of $4 \mathrm{M} \mathrm{NaOH}$ solution, during the whole process. The fed-batch fermentation cycle was $168 \mathrm{~h}$. During fermentation, glucose concentration in the broth was maintained at 30-80 g/L. Rapeseed oil supplementation rate was $0.5 \mathrm{~g} / \mathrm{L} / \mathrm{h}$ for the first $24 \mathrm{~h}$, after which rapeseed oil concentration was maintained at 2-15 g/L, according to rapeseed oil consumption. The semi-continuous fermentation period was $378 \mathrm{~h}$, and SLs was intermittently separated by the separation unit every $72 \mathrm{~h}$. The supplementation rate of glucose and rapeseed oil was optimized according to the sedimentation rate of SLs. After every two in-situseparations, $1 / 5$ of the initial nutrition was supplemented.

\subsection{SLs sedimentation mechanism}

\subsubsection{Influences of rapeseed oil to SLs ratios on SLs morphology and sedimentation}

The ratio of rapeseed oil to SLs $(\mathrm{O} / \mathrm{S})(0.075,0.10,0.13,0.15,0.18,0.20,0.22,0.23,0.25,0.30 \mathrm{~g} / \mathrm{g})$ was quickly detected and regulated by the low-field nuclear magnetic method. ${ }^{[19]}$ SLs morphology was observed by optical microscopy (BMDH200 microscope, Sunny Optical Technology Co., Ltd), and the main structures of SLs with different morphologies were analyzed by LC-MS descripted as Chen. ${ }^{[7]}$

Furthermore, $10 \mathrm{~mL}$ of the fermentation broth with different $\mathrm{O} / \mathrm{S}$ ratios were processed by standing still to observe the SLs sedimentation, and the volume of lower layer was recorded at different time points until it was unchanged during $2 \mathrm{~h}$.

\subsubsection{Measurements of broth viscosity and SLs particle size}

Twenty milliliters of broth with different $\mathrm{O} / \mathrm{S}$ ratios were obtained to measure the viscosity of supernatant with a viscosity meter (Brookfield DV-II). Another $5 \mathrm{~mL}$ broth was thoroughly mixed, and the SLs particle size was analyzed by nanometer size and ZETA potential analyzer (Beckman Coulter Nano-ZS).

\subsection{Effect of ultrasound assistance on SLs precipitation}

2.3.1. Effect of ultrasonic ultrasound assistance on cell activity, SLs production, bubble and particle size

An appropriate amount of fermentation broth was obtained and treated at different ultrasonic times $(0$, $10,20,30,40,50$, and $60 \mathrm{~min})$ and power $(100,200$, and $300 \mathrm{~W})$ in a $10 \mathrm{~L}$ ultrasonic cleaning machine (SHT-70al, $40 \mathrm{kHz}$ ). And then, the treated broth was diluted at appropriate times and coated on the plate to observe the colony-forming unit (CFU) after $72 \mathrm{~h}$. Otherwise, after different ultrasonic treatments, the 
cells from $50 \mathrm{~mL}$ fermentation broth were concentrated by centrifugation, resuspended, and inoculated into a medium only containing glucose and rapeseed oil in a $500 \mathrm{~mL}$ shake flask with $50 \mathrm{~mL}$ working volume to investigate the SLs production capacity.

An appropriate amount of fermentation broth was obtained and treated at $100 \mathrm{~W}$ power in a $10 \mathrm{~L}$ ultrasonic cleaning machine, and record the level of fermentation broth. In addition, An appropriate amount of fermentation $\operatorname{broth}(\mathrm{O} / \mathrm{S}=0.1)$ was obtained and treated at different ultrasonic power $(0,100,200$, and 300 W) for $5 \mathrm{~s}$, and detected particle size.

\subsection{Semi-continuous fermentation based on ultrasonic assisted treatment}

In semi-continuous fermentation, the optimal $\mathrm{O} / \mathrm{S}$ ratio was adopted to rational control the sedimentation of SLs for in-situ separation. According to our previously developed SLs separation equipment, the improved in-situ separation platform of UEST was shown in Fig 1. ${ }^{[20]}$ The fermentation broth was rapidly pumped into the separation unit, which was placed in the ultrasonic machine, by a peristaltic pump at $5 \mathrm{~mL} / \mathrm{s}$. The ultrasonic power and time were controlled at $100 \mathrm{~W}$ and $10 \mathrm{~min}$. Subsequently, the SLs was intermittently discharged from port $\mathrm{c}$ for $1 \mathrm{~min}$, and the supernatant was refilled from port $\mathrm{b}$ to the fermenter until the SLs concentration in the fermenter was lower than $60 \mathrm{~g} / \mathrm{L}$. Finally, the separated crude SLs was washed by $1 / 2$ proportion of sterile water $(\mathrm{v} / \mathrm{v})$, and thoroughly mixed by stirring. After standing for 20 min, the supernatant was pumped back to the fermenter to recover the biomass, glucose and rapeseed oil. Due to the loss of biomass after the operation of SLs in-situ separation, medium and sterile water were replenished to the initial volume of $2.5 \mathrm{~L}$.

\subsection{Analytical methods}

Glucose concentration was analyzed by an enzymatic bio-analyzer (SBA-40C, Shandong Academy of Sciences, China). Rapeseed oil concentration in the broth was quickly determined by the low-field nuclear magnetic method. ${ }^{[19]}$ For biomass determination, three parallel broth samples were washed twice with alcohol and dried at 80 for $24 \mathrm{~h}$ to measure dry cell weight (DCW). The concentrations and structures of SLs were determined by HPLC and HPLC-MS, respectively. ${ }^{[7]}$

\subsection{Data analysis}

As the fermentation went on, SLs was gradually accumulated in the broth and once the $\mathrm{O} / \mathrm{S}$ ratio reached at a certain range, the SLs sedimentation was occurred. The average sedimentation rate was calculated by formula (1). By fitting the height of SLs sedimentation was directly proportional to the concentration of SLs, formula (2) was obtained. The sedimentation rate formula related to SLs concentration could be derived in (3).

$$
\begin{aligned}
& V=\frac{H_{1}-H_{2}}{2 t}(1) \\
& \frac{H_{2}}{H_{1}}=0.0031 \times c-0.0001(2) \\
& V=\frac{H_{1}-H_{1}(0.0031 \times c-0.0001)}{2 t}(3)
\end{aligned}
$$

Where $V$ is the average sedimentation rate of SLs in $\mathrm{cm} / \mathrm{s}, \mathrm{H}_{1}$ is the height of broth in the $10 \mathrm{~mL}$ sedimentation tube in $\mathrm{cm}, \mathrm{H}_{2}$ is the height of SLs settled in the $10 \mathrm{~mL}$ sedimentation tube in $\mathrm{cm}$, $\mathrm{t}$ is settling time in $\mathrm{s}, \mathrm{c}$ is SLs concentration in $\mathrm{g} / \mathrm{L}$.

Due to the consideration of working volume change by feeding, all the presented data in Figures and Tables were normalized to the initial volume. All experiments were performed in triplicate.

\section{Results}

3.1. Effects of $\mathrm{O} / \mathrm{S}$ ratios on SLs morphology and sedimentation

Under different $\mathrm{O} / \mathrm{S}$ ratio conditions, SLs would exhibit different morphologies. When the $\mathrm{O} / \mathrm{S}$ value was higher than $0.04 \mathrm{~g} / \mathrm{g}$, the SLs presented noncrystalline characteristics, definition of oily SLs. The microscopic 
results showed that biomass and broth were enclosed by the mixture of rapeseed oil and SLs due to their strong hydrophobicity. Under the action of gravity, SLs began to gradually precipitate, and the fermentation broth showed obvious stratification (Fig. 2). With the decrease of $\mathrm{O} / \mathrm{S}$ ratio, the fermentation broth began to be in an emulsified state, and SLs no longer stratified with fermentation broth, thus presenting a relatively uniform state (Fig. 2). With the further decrease of $\mathrm{O} / \mathrm{S}$ ratio $(<0.02 \mathrm{~g} / \mathrm{g})$, crystalline SLs gradually appeared in the broth (Fig. 2). By comparing the structure compositions of SLs in different forms, it could be found that under high O/S ratio condition $(>0.04 \mathrm{~g} / \mathrm{g})$, the proportion of deposited SLs, which was mainly a mixture of lactone- and acidic-forms SLs, accounted for $92 \%$ of the total SLs, while the supernatant only contained a small portion of lactone- and acidic- form SLs. In contrast, when the O/S ratio was low $(<$ $0.02 \mathrm{~g} / \mathrm{g}$ ), the crystalline SLs composed of lactone-form SLs accounted for $38 \%$ of the total SLs, whereas the broth consisted of lactone- and acidic-form SLs (Table S1). These results were in agreed with the conclusion that acidic-form SLs has stronger hydrophilicity and lactone-form SLs has stronger hydrophobicity. Notably, the crystalline SLs could not settle on its own, but as the O/S ratio increased, it could be found through the microscope that the crystalline SLs would be surrounded by oily particles to form oily SLs, and then began to precipitate (Fig. S1). Therefore, it was further inferred that the settleability of SLs was dependent on their morphologies, which could be contributed to the hydrophobicity of lactone-form SLs as well as the rapeseed oil and SLs contents.

\subsection{The characteristic of SLs sedimentation}

When the $\mathrm{O} / \mathrm{S}$ ratio was greater than $0.04 \mathrm{~g} / \mathrm{g}$, the sedimentation height was directly proportional to the concentration of SLs (Fig. 3A), and then the average sedimentation rate could be obtained by formula (1)-(3). According to formula (3), although the average sedimentation rate was related to SLs concentration in the broth, the rapeseed oil content would significantly affect the settling time, thus exhibiting that $\mathrm{O} / \mathrm{S}$ ratio was correlated to the average sedimentation rate (Fig. 3B). With the increase of $\mathrm{O} / \mathrm{S}$ ratio, the average of sedimentation rate of SLs was significantly enhanced, which reached the maximum value of $0.075 \mathrm{~cm} / \mathrm{s}$ at $0.25 \mathrm{~g} / \mathrm{g}$. However, it started to decrease with the further increase of $\mathrm{O} / \mathrm{S}$ ratio. As the main reason was the increase of rapeseed oil concentration, the density of the mixture of SLs and rapeseed oil was lower. Therefore, the average of sedimentation rate of SLs was decreased. Further increase of O/S ratio can achieve temporary suspension, but SLs would be re-sedimented by standing still.

It was found that the $\mathrm{O} / \mathrm{S}$ ratio was the key factor affecting the morphology and subsequent settling of SLs. In general, the sedimentation rate of mixture (SLs and rapeseed oil) particles was mainly determined by the radius, density, and viscosity. Analysis of SLs particle size showed that when SLs was oily, with the increased of $\mathrm{O} / \mathrm{S}$ ratio, the particle size of the mixture gradually increased, and the maximum radius was about $3.0 \mathrm{~mm}$ (Fig. 3C). Therefore, the particle size of mixture may be an important factor affecting on the SLs settling. In the early stage of fermentation, the viscosity of supernatant had marginal changes. When the SLs concentration was higher than $250 \mathrm{~g} / \mathrm{L}$, the supernatant viscosity was sharply increased (Fig. 3D). The high viscosity would affect the mixing and mass transfer, and reduce the production efficiency of SLs in the late fermentation. Therefore, when SLs concentration was around 200-220 g/L, the in-situ separation would be carried out in this study, thus viscosity had limited influence on SLs precipitation. By the density analysis, it was shown that after mixing SLs and rapeseed oil, the density decreased with the increase of O/S ratio (Fig. 3E). However, $1.0 \mathrm{~g}$ SLs was found to be bound to a maximum of $0.3 \mathrm{~g}$ rapeseed oil (Fig. S2). Therefore, the minimum density of the mixture of SLs and rapeseed oil was $1.10 \mathrm{~g} / \mathrm{cm}^{3}$. In contrast, the density of the supernatant was mainly determined by glucose concentration, which was less than $100 \mathrm{~g} / \mathrm{L}$ in real fermentation process, so the density of the supernatant was much lower than the mixture of SLs and rapeseed oil (Fig. 3F), and density difference was the main reason determining the SLs settling or floating. When the $\mathrm{O} / \mathrm{S}$ ratio was low $(<0.04 \mathrm{~g} / \mathrm{g})$, crystallized or emulsified SLs would not settle by itself. When the $\mathrm{O} / \mathrm{S}$ ratio was high $(>0.04 \mathrm{~g} / \mathrm{g})$, an oily mixture was formed and began to settle. With the further increase of $\mathrm{O} / \mathrm{S}$ ratio, the hydrophobicity of the mixture was strengthened and the particle size increased, thus the settling rate was accelerated under gravity action.

In the settling process, the hydrophobic mixture formed by SLs and rapeseed oil was the key to settling (Fig. 
S3). The stronger hydrophobicity, the larger the particle size, the faster sedimentation rate will be. However, with the further increased of $\mathrm{O} / \mathrm{S}$ ratio, density became the main influencing factor, so that the sedimentation rate of mixture decreased or suspension. In terms of sedimentation rate, it was mainly dependent on the particle size of mixture and density difference. To improve the efficiency of SLs separation and reduce the loss of substances during in-situ separation process, low glucose concentration and appropriate $\mathrm{O} / \mathrm{S}$ ratio should be adopted.

\subsection{Enhancement of sedimentation efficiency by UEST}

Although SLs sedimentation could be achieved by adjusting the $\mathrm{O} / \mathrm{S}$ ratio in the broth, it was common to result in some losses of biomass, glucose, and rapeseed oil during the in-situ separation process. Especially for rapeseed oil, its loss and SLs sedimentation efficiency always presented a contradiction, and it would be lost a lot at high $\mathrm{O} / \mathrm{S}$ ratio of $0.25 \mathrm{~g} / \mathrm{g}$, even if SLs could be quickly settled. On the other hand, under the low $\mathrm{O} / \mathrm{S}$ ratio condition, the rapeseed oil loss was reduced, but corresponding SLs sedimentation rate also slowed down. Therefore, UEST was introduced to accelerate the sedimentation rate of SLs and simultaneously reduce the loss of substrate and biomass. Ultrasound not only accelerates the aggregation of SLs particles, enhancing the gravity force, but also rapidly removes air bubbles, reducing the interference of air bubbles on SLs precipitation (Fig. S4 A). By comparing the effect of ultrasonic on particle size, it can be found that ultrasonic enlarged the particle size of mixture, increasing by more than $37.3 \%$ (Fig. S4 B). Moreover, it could be found that after the treatment of ultrasound, the cell viability and SLs production capability would not be affected (Fig. S4 C and D). Therefore, ultrasound can accelerate the deposition of SLs by reducing bubbles and increasing particle size, and has no significant effect on the growth of strain and SLs synthesis.

The average sedimentation rate of SLs enhanced with the increase of the ultrasonic time and power (Fig. 4A, $\mathrm{B}$ and $\mathrm{C}$ ). The maximum sedimentation rate increases by $46.9 \%$ to $485.4 \%$ with UEST from high to low O/S ratios (Fig. $4 \mathrm{D}$ ). When the $\mathrm{O} / \mathrm{S}$ was $0.10 \mathrm{~g} / \mathrm{g}$, the sedimentation rate reached $0.0165 \mathrm{~cm} / \mathrm{s}$, independent on ultrasonic power. Since low O/S ratio leaded to less loss in in-situ separation process, UEST could not only reduces the influence of rapeseed oil on viscosity, but also further reduces the loss of rapeseed oil and accelerates SLs separation under low $\mathrm{O} / \mathrm{S}$ ratio condition. In the following experiments, the ultrasonic power of $100 \mathrm{~W}$ and the time of $10 \mathrm{~min}$ (the height of separation device was $10 \mathrm{~cm}$ and the sedimentation rate was $0.0165 \mathrm{~cm} / \mathrm{s}$ ) were adopted to achieve effective sedimentation.

\subsection{Semi-continuous fermentation of SLs production by in-situ separation strategy with UEST}

The SLs sedimentation was regulated by the S/O ratio. Therefore, before in-situ separation of SLs, the $\mathrm{O} / \mathrm{S}$ ratio was adjusted to $0.10-0.12 \mathrm{~g} / \mathrm{g}$, and the glucose concentration was controlled at approximately 30 $\mathrm{g} / \mathrm{L}$. The whole semi-continuous fermentation cycle lasted $378 \mathrm{~h}$, during which 4 times of in-situ separation of SLs were conducted with UEST once the SLs concentration in the broth reached 200-220 g/L, and finally the total of $2039.9 \mathrm{~g}$ SLs was produced with the consumption of $1545.7 \mathrm{~g}$ rapeseed oil and $1979.3 \mathrm{~g}$ glucose respectively (Fig. 5A and B). Through the analysis of SLs productivity and yield during different phases, it was found that the cell activity has been maintained at a high level throughout the whole fermentation process and the average SLs productivity and yield reached $2.15 \mathrm{~g} / \mathrm{L} / \mathrm{h}$ and $0.58 \mathrm{~g} / \mathrm{g}$ respectively (Fig. 5C). Moreover, UEST in-situ separation could reduce the losses of biomass, glucose and rapeseed oil by $68.2 \%$, $16.2 \%$, and $65.5 \%$, respectively (Table 1), in comparison to direct in-situseparation strategy without UEST, thus achieving efficient SLs separation under low $\mathrm{O} / \mathrm{S}$ ratio condition. Correspondingly, thein-situseparation efficiency and SLs separation rate improved by $34.5 \% \%$ and $26.4 \%$, respectively (Table 1 ).

\section{Discussion}

SLs has been regarded as a green and sustainable biosurfactants, but their high production costs limit largescale application and development. ${ }^{[21-23]}$ Coupling fermentation on the basis of in-situ product separation is one of the most effective ways to reduce production costs and improve productivity (Table 2). Wang designed a simple in-situ separation device coupled with the bioreactor and adopted high cell density fermentation to achieve high-efficient production of SLs. ${ }^{[24]}$ After running for $480 \mathrm{~h}$, the productivity and yield of SLs were $2.39 \mathrm{~g} / \mathrm{L} / \mathrm{h}$ and $0.73 \mathrm{~g} / \mathrm{g}$, respectively, with an average separation efficiency of $74.3 \%$. Zhang developed a new 
bioreactor with dual ventilation pipes containing dual sieve plates (DVDSB) for semi-continuous fermentation of SLs. ${ }^{[15]}$ By regulating the air pressure and different sources of oil (transgenic or non-transgenic oil), the gravity sedimentation of SLs at the bottom of bioreactor could be accomplished with SLs titer, productivity, and yield of $477 \mathrm{~g} / \mathrm{L}, 1.59 \mathrm{~g} / \mathrm{L} / \mathrm{h}$ and $0.60 \mathrm{~g} / \mathrm{g}$, respectively. Otherwise, Dolman suggested that the glucose concentration could adjust the density of fermentation broth thereby controlling the ups and downs of SLs. ${ }^{[25]}$ The whole fermentation process lasted $1023 \mathrm{~h}$ to produce $623 \mathrm{~g} / \mathrm{L}$ SLs, and during the SLsinsitu separation process, the recovery rate of SLs was as high as $86.0 \%$, which was 9 times concentration of previously reported. Apart from SLs separation by gravity sedimentation, Liu realized the SLs separation in the upper layer of the broth through the principle of froth flotation. Subsequently, part of biomass and glucose could be recovered by washing the settled SLs, and the final titer and productivity of SLs were 342 $\mathrm{g} / \mathrm{L}$ and $1.55 \mathrm{~g} / \mathrm{L} / \mathrm{h}$ for $221 \mathrm{~h}$, respectively. ${ }^{[20]}$ Though these strategies have achieved relatively satisfactory results in the in-situ separation of SLs, the mechanism of SLs separation either by gravity sedimentation or by froth flotation was still not been clearly elucidated, which led to a blind separation process and low separation efficiency. Oil or glucose concentration has been pointed out to possess a significant impact on the settlement of SLs. ${ }^{[15]}$ However, these researches focused more on the final results and failed to further analyze the causes of the sedimentation, resulting in big differences in different studies.

Herein, the sedimentation mechanism of SLs was discussed and it was found that the $\mathrm{O} / \mathrm{S}$ ratio was the key factor affecting the morphology and subsequent settling of SLs. In terms of sedimentation rate, dependent on the particle size of mixture and density difference. To improve the efficiency of SLs separation and reduce the loss of substances during in-situ separation process, low glucose concentration and appropriate O/S ratio should be adopted. On the other hand, the UEST can accelerate the particle collision, make the particle become larger, eliminate the air bubbles, and further reduces the settling resistance. ${ }^{[26]}$ Palme and Hincapie Gomez applied ultrasonic enhanced deposition technology in the separation and recovery of microalgae and yeast cells, respectively. ${ }^{[16,18]}$ In this work, UEST was introduced to enhance the sedimentation of SLs, simultaneously greatly reduce the losses of rapeseed oil, glucose, and biomass duringin-situ separation process. Moreover, semi-continuous fermentation by in-situ separation of SLs with UEST was conducted. Compared to the batch fermentation $(168 \mathrm{~h})$, the fermentation cycle was more than 2-fold longer $(378 \mathrm{~h})$, and the average productivity and yield from semi-continuous fermentation were increased by $26.5 \%$ and $23.4 \%$ respectively. This could be contributed to that the semi-continuous fermentation could separate out SLs and toxic substances in time, and allow the cells being in the optimal production environment, which also proved the feasibility of semi-continuous fermentation as a model for the efficient production of SLs. In comparison with other semi-continuous fermentations of SLs, both the productivity and yield in this work were at high levels, especially for the index of $\mathrm{Y}_{\mathrm{SLs} / \mathrm{DCW}}$ representing the SLs production yield to the $\mathrm{DCW}$, its value reached $36.1 \mathrm{~g} / \mathrm{g}_{\mathrm{DCW}}$, which was $86.1 \%$ higher than the highest value in the literatures. Furthermore, the specific SLs productivity $\left(\mathrm{Q}_{\mathrm{P} / \mathrm{DCW}}\right)$ also exhibited a $41.2 \%$ higher than the highest value ever reported. It could be expected to achieve a more ideal production efficiency in combination with high cell density strategy, laying a foundation for further industrial application.

\section{Conclusion}

In this study, the mechanism of SLs sedimentation in multiphase fermentation process was elucidated for the ratio of $\mathrm{O} / \mathrm{S}$. And then UEST was introduced to strengthen the SLs sedimentation rate. The real-time rational in-situ separation of SLs and semi-continuous fermentation with UEST was accomplished to achieve productivity and yield of $2.15 \mathrm{~g} / \mathrm{L}$ and $0.58 \mathrm{~g} / \mathrm{g}$ respectively as well as reduce the losses of cells, glucose, and rapeseed oil by $68.2 \%, 16.2 \%$, and $65.5 \%$, respectively. The rational in-situ separation and UEST for complex multiphase systems could be extended to other biosurfactant fermentation systems to improve production efficiency.

\section{Declarations}

\section{Acknowledgement and Funding}

This work was financially supported by the National Key Research Development Program of China (No. 
2019YFA0904803). The authors thank Research Center of Analysis and Test of East China University of Science and Technology for the help on the characterization.

\section{Competing interests}

The authors declare that they have no competing interests.

\section{Availability of data and materials}

All data generated or analyzed during this study are included in this published article.

\section{Author Contribution Statement}

CY: Conceptualization, Methodology, Validation, Formal analysis, Investigation, Writing - original draft \& editing, Visualization.

TX, LY, LC: Conceptualization, Methodology.

CJ, ZYP, TXW: Conceptualization, Validation, Writing - review \& editing, Supervision, Funding acquisition.

\section{Ethical approval}

This article does not contain any studies with human participants or animals performed by any of the authors.

\section{Consent for participate}

Not applicable.

\section{Consent for publication}

Written informed consent for publication was obtained from all participants.

\section{Reference}

[1] R. Jahan, A. M. Bodratti, M. Tsianou, P. Alexandridis, Adv. Colloid. Interfac. 2020 , 275, 102061. https://doi.org/10.1016/j.cis.2019.102061.

[2] I. N. A. Van Bogaert, K. Saerens, C. De Muynck, D. Develter, W. Soetaert, E. J. Vandamme, Appl. Microbiol. Biot. 2007, 76, 23.

[3] V. K. Gaur, R. K. Regar, N. Dhiman, K. Gautam, J. K. Srivastava, S. Patnaik, M. Kamthan, N. Manickam, Bioresource Technol.2019, 285, 121314.

[4] J. Thaniyavarn, T. Chianguthai, P. Sangvanich, N. Roongsawang, K. Washio, M. Morikawa, S. Thaniyavarn, Biosci. Biotech. Bioch.2008, 72, 2061.

[5] S. Sen, S. N. Borah, A. Bora, S. Deka, Microb. Cell Fact.2017 , 16, 95. https://doi.org/10.1186/s12934017-0711-z.

[6] G. Zhou, X. W. Tian, Y. M. Lin, S. L. Zhang, J. Chu, Bioproc. Biosyst. Eng. 2019 , 42, 575.

[7] Y. Chen, X. W. Tian, Q. H. Li, Y. Li, J. Chu, H. F. Hang, Y. P. Zhuang, Bioresource Technol. 2020 , $315,123586$.

[8] A. C. David, G. C. David, J. Biotechnol. 2003, 103, 31.

[9] Y. M. Hu, L. K. Ju, Enzyme Microb. Tech. 2001 , 29, 593.

[10] P. Jimnez-Pealver, A. Koh, R. Gross, T. Gea, X. Font, J. Surfactants Deterg. 2020 , 23, 481.

[11] R. D. Ashby, A. J. McAloon, D. K. Y. Solaiman, W. C. Yee, M. Reed, J. Surfactants Deterg. 2013, 16, 683. 
[12] X. J. Ma, L. Meng, H. M. Zhang, L. Y. Zhou, J. Y. Yue, H. X. Zhu, R. S. Yao, Appl. Microbiol. Biot. $2020,104,77$.

[13] X. Q. Jia, L. Qi, Y. G. Zhang, X. Yang, H. N. Wang, F. L. Zhao, W. Y. Lu, Chinese J. Chem. Eng. $\mathbf{2 0 1 7}, 25,732$.

[14] H. M. Wang, S. L. K. W. Roelants, M. H. To, R. D. Patria, G. Kaur, N. S. Lau, C. Y. Lau, I. N. A. Van Bogaert, W. Soetaert, C. S. K. Lin, J. Chem. Technol. Biot. 2019, 94, 999. https://doi.org/10.1002/jctb.5847.

[15] Y. G. Zhang, D. Jia, W. Q. Sun, X. Yang, C. B. Zhang, F. L. Zhao, W. Y. Lu, Microb. Biotechnol.2018 , 11, 455 .

[16] E. Hincapie Gomez, A. J. Marchese, Biotechnol. Progr.2015, 31, 414.

[17] G. L. Maddikeri, P. R. Gogate, A. B. Pandit, Chem. Eng. J. 2015 , 263, 479.

[18] O. Palme, G. Comanescu, I. Stoineva, S. Radel, E. Benes, D. Develter, V. Wray, S. Lang, Eur. J. Lipid Sci. Tech.2010, 112, 663.

[19] Y. Chen, Y. M. Lin, X. W. Tian, Q. H. Li, J. Chu, J. Microbiol. Meth. 2019 , 157, 9.

[20] Z. P. Liu, X. W. Tian, Y. Chen, Y. M. Lin, A. Mohsin, J. Chu,Process Biochem. 2019, 81, 1.

[21] P. Jimenez-Penalver, A. Rodriguez, A. Daverey, X. Font, T. Gea,Rev. Environ. Sci. Bio. 2019, 18, 413.

[22] S. Lodens, S. L. K. W. Roelants, K. Ciesielska, R. Geys, E. Derynck, K. Maes, F. Pattyn, L. Van Renterghem, L. Mottet, S. Dierickx, L. Vanhaecke, B. Devreese, S. L. De Maeseneire, W. Soetaert,Biotechnol. Bioeng.2020 , 117, 453.

[23] L. Van Renterghem, S. L. K. W. Roelants, N. Baccile, K. Uyttersprot, M. C. Taelman, B. Everaert, S. Mincke, S. Ledegen, S. Debrouwer, K. Scholtens, C. Stevens, W. Soetaert, Biotechnol. Bioeng. 2018 , 115, 1195 .

[24] H. M. Wang, G. Kaur, M. H. To, S. L. K. W. Roelants, R. D. Patria, W. Soetaert, C. S. K. Lin, J. Clean Prod. $2020,246,118995$.

[25] B. M. Dolman, C. Kaisermann, P. J. Martin, J. B. Winterburn,Process Biochem. 2017 , 54, 162.

[26] X. M. Luo, H. Y. Gong, J. H. Cao, H. R. Yin, Y. P. Yan, L. M. He, Chem. Eng. Sci. 2019 , 203, 285.

Figure captions

Fig. 1.Semi-continuous fermentation platform (a. Fermentation broth inlet; b. Supernatant outlet; c. SLs export).

Fig. 2. The morphology and sedimentation of SLs at different $\mathrm{O} / \mathrm{S}$ ratios.

Fig. 3. SLs precipitation. (A) Relationship between the sedimentation height ratio of SLs and the concentration; (B) Relationship between the average sedimentation rate of SLs and O/S; (C) SLs particle size; (D) Viscosity of fermentation supernatant; (E) Density of SLs and oil mixture; (F) Density of fermentation broth supernatant.

Fig. 4. Ultrasonic assisted SLs deposition. (A) $100 \mathrm{~W}$ ultrasonic power; (B) $200 \mathrm{~W}$ ultrasonic power; (C) $300 \mathrm{~W}$ ultrasonic power; (D) Maximum sedimentation rate at different power.

Fig. 5.Ultrasound-assisted in-situseparation. (A) SLs titer; (B) Residual oil, glucose and DCW concentration; (C) Productivity and yield of SLs.

Fig. 1 


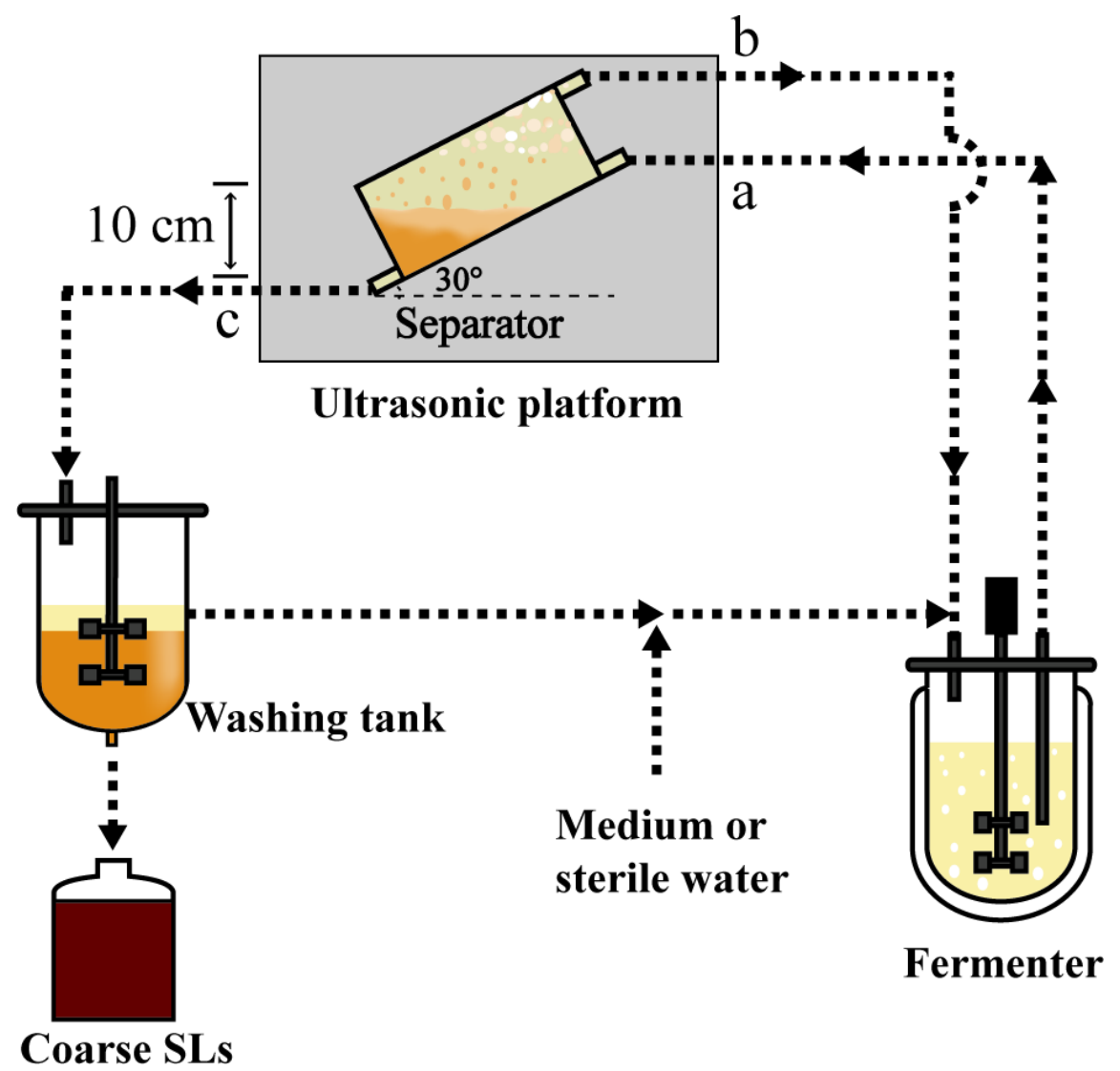

Fig. 2

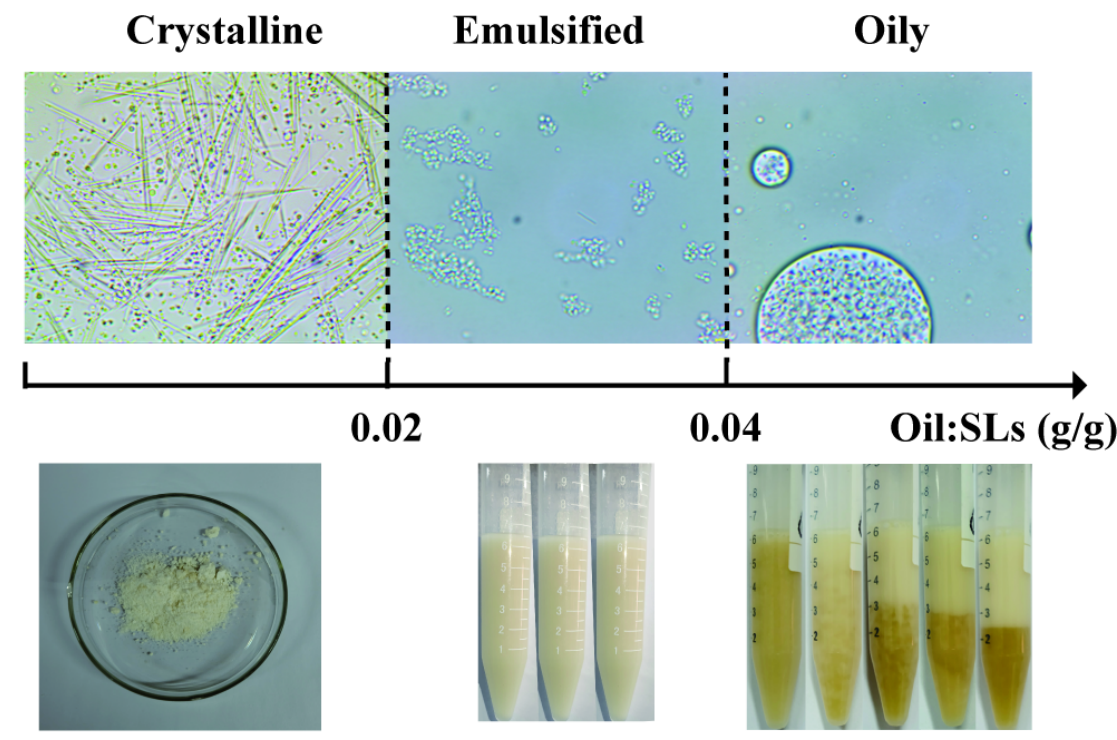

Cooling crystallization

Gravity precipitation

Fig. 3 

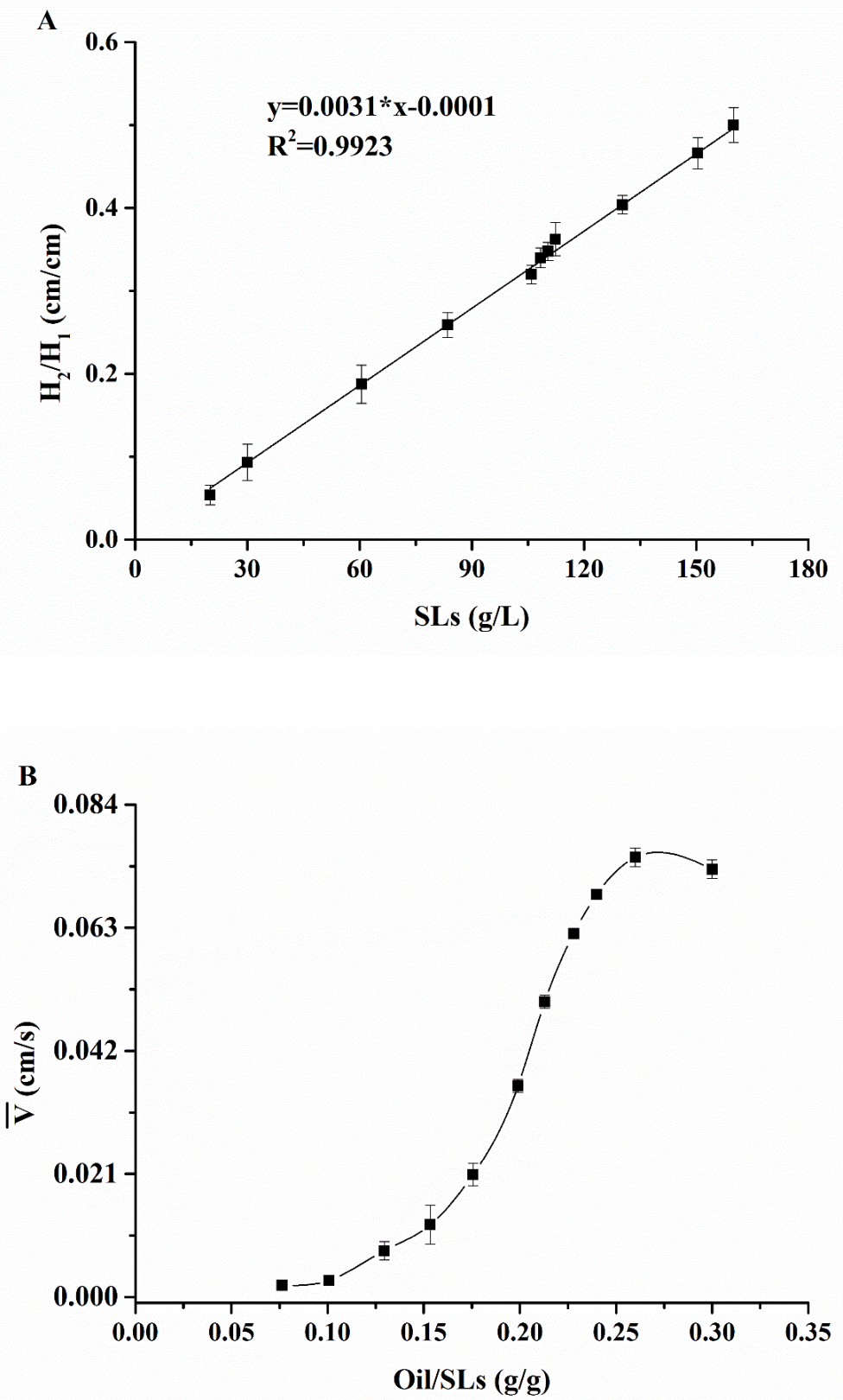

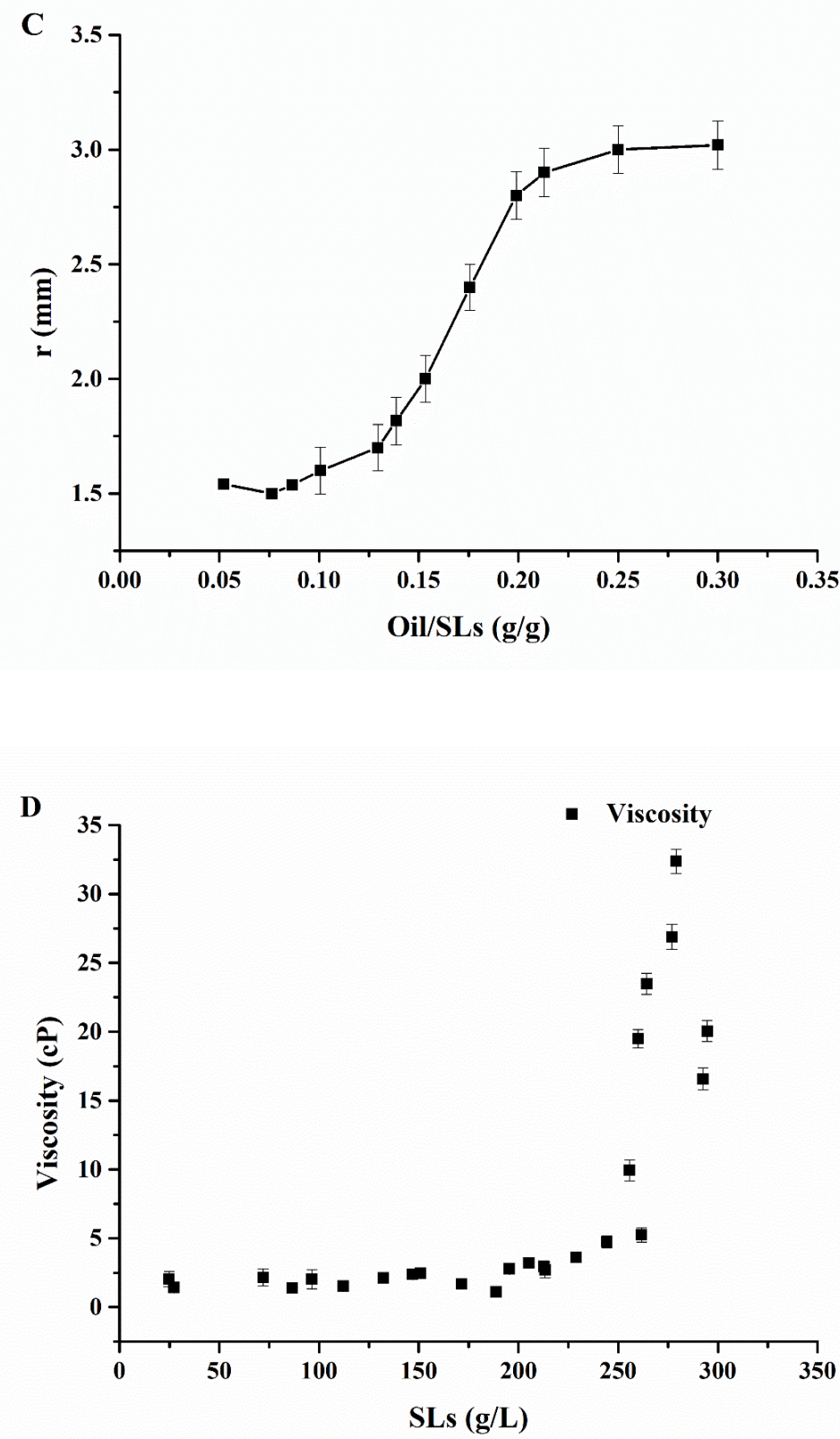

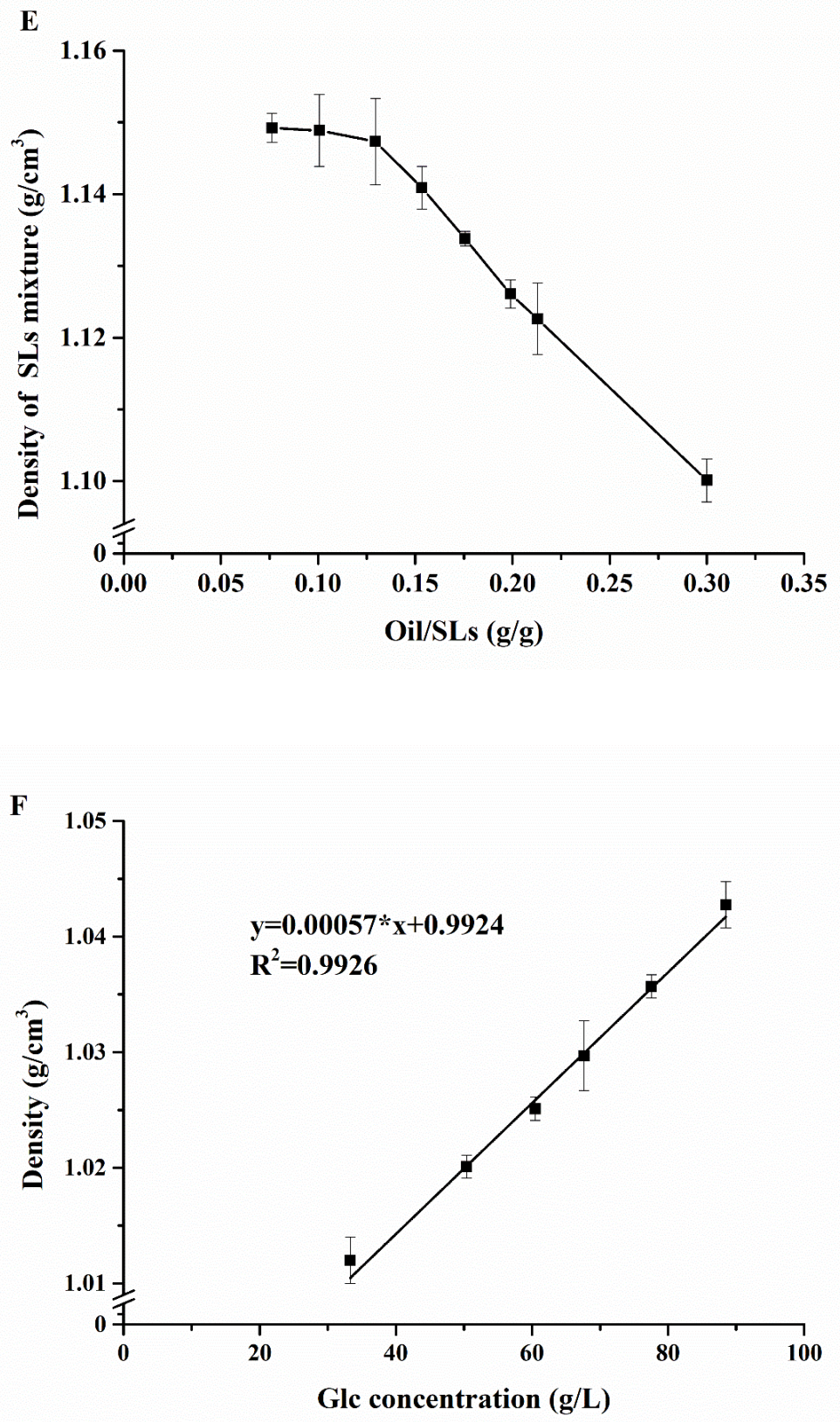

Fig. 4 

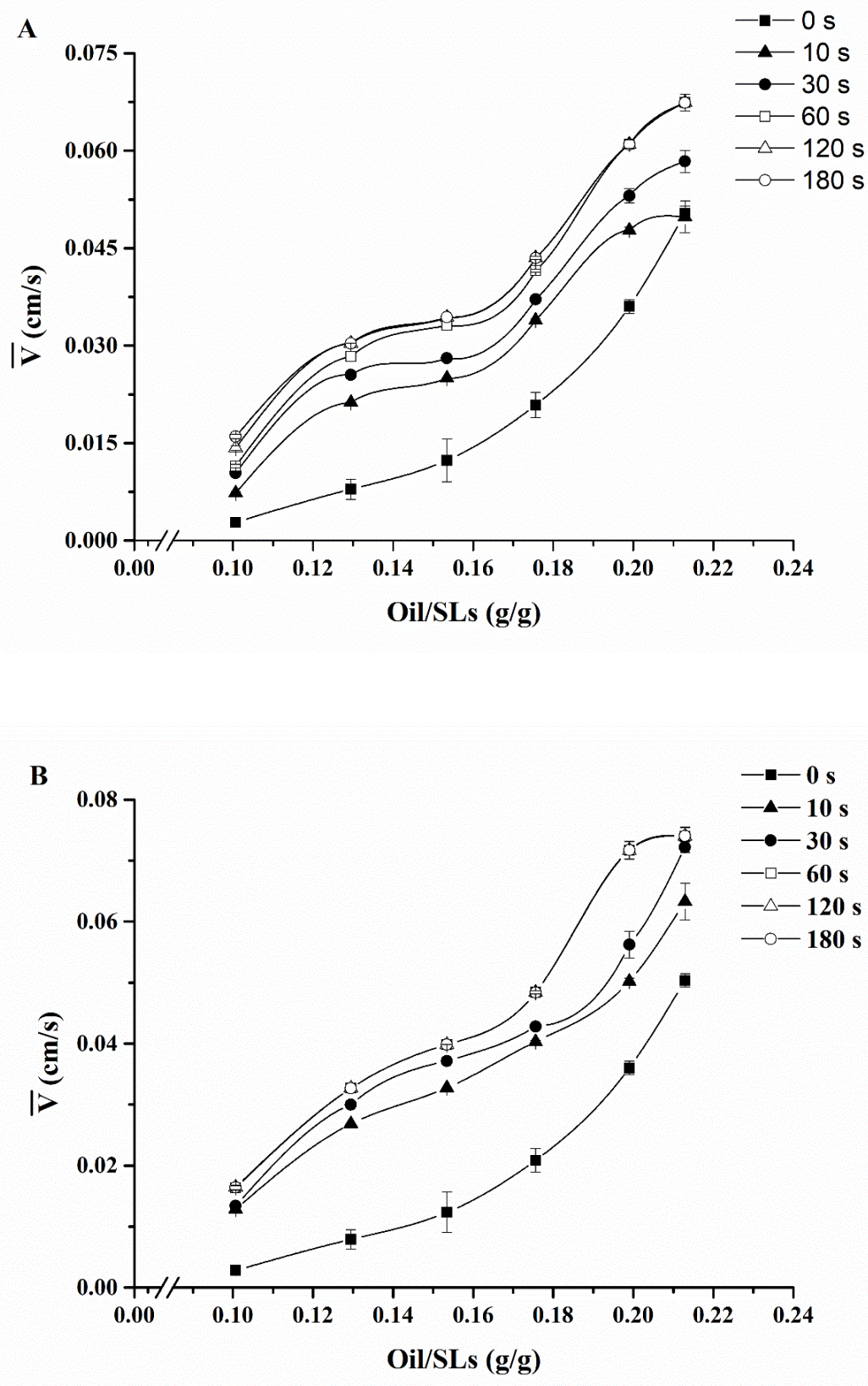

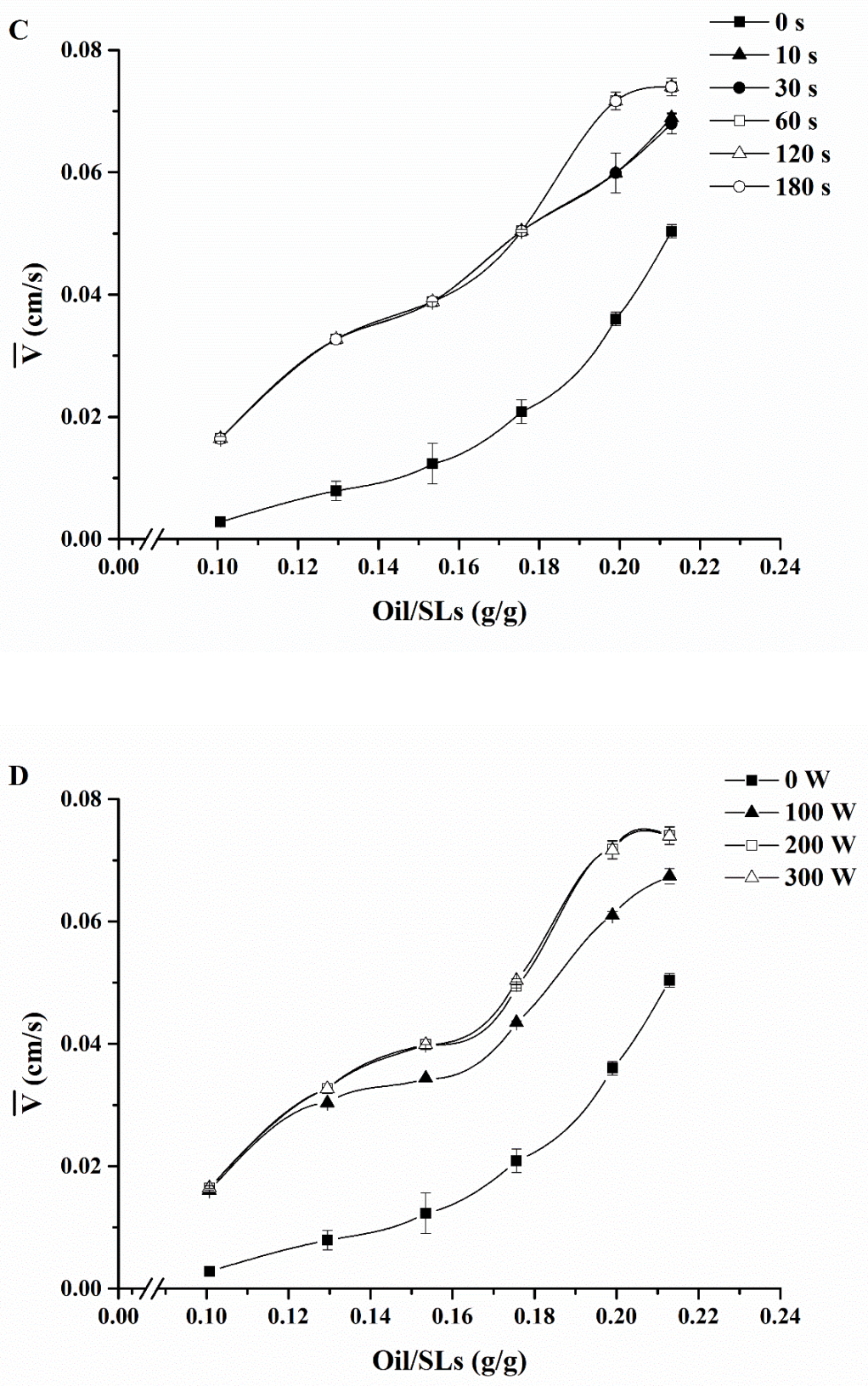

Fig. 5 

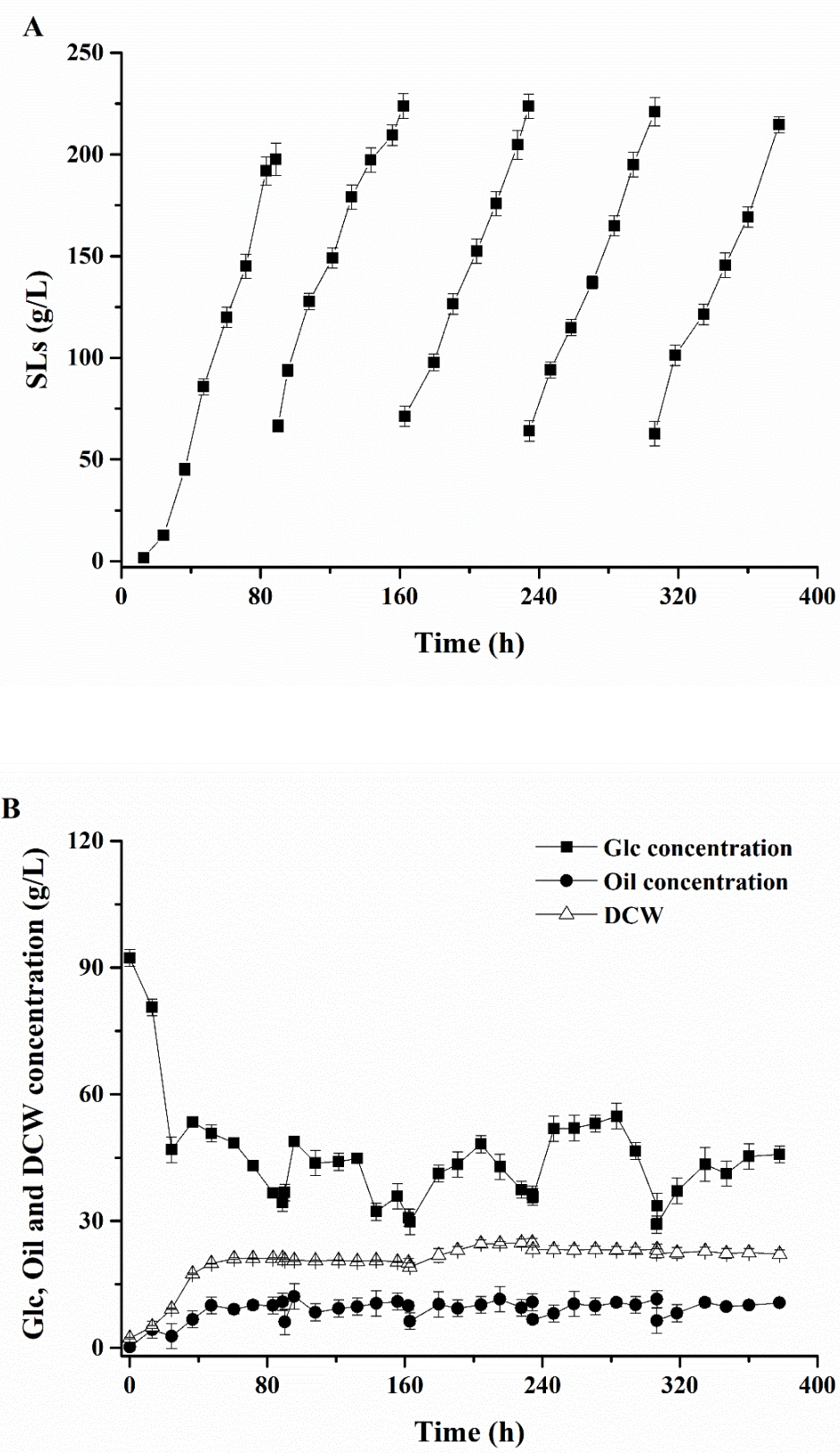


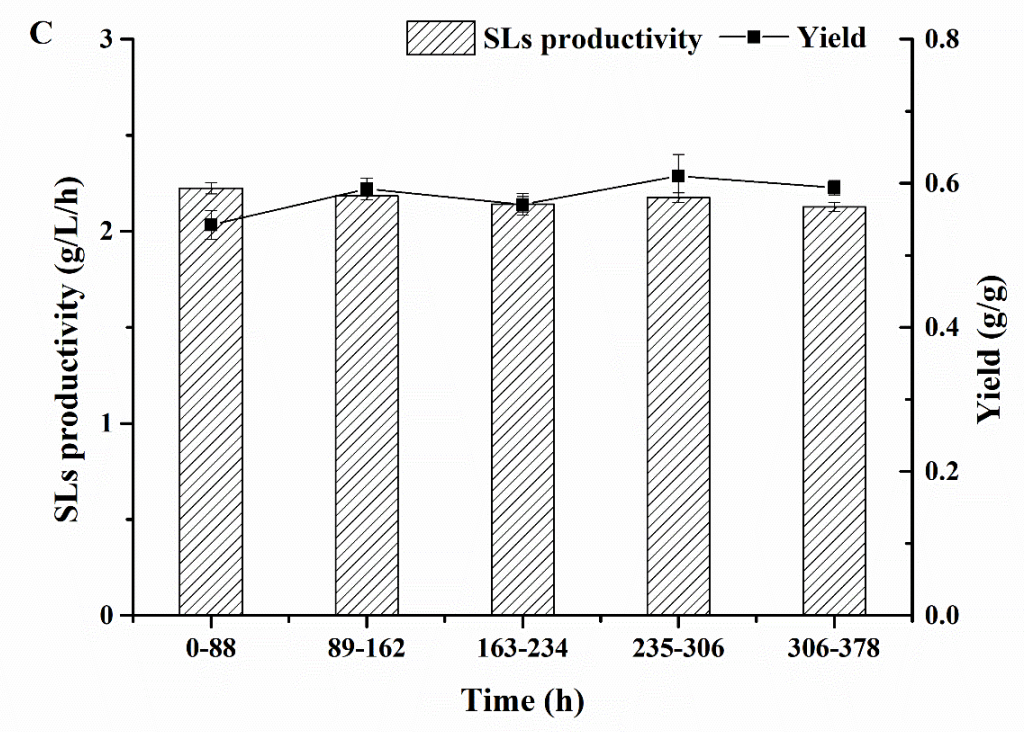

Table 1 In-situseparation parameter

\begin{tabular}{llllll}
\hline Model & Biomass loss rate $(\%)^{\mathrm{a}}$ & ${\text { Glucose loss rate }(\%)^{\mathrm{a}}}$ & ${\text { Oil loss rate }(\%)^{\mathrm{a}}}$ & ${\text { SLs separation rate }(\%)^{\mathrm{b}}}$ & $I n-s i$ \\
\hline UEST & $0.14 \pm 0.024$ & $5.70 \pm 0.15$ & $3.90 \pm 0.31$ & 71.4 & 7.52 \\
In-situ separation & $0.44 \pm 0.010$ & $6.80 \pm 0.02$ & $11.30 \pm 0.33$ & 56.5 & 5.59 \\
\hline
\end{tabular}

aBiomass (Glucose, oil) los rate $=\frac{c_{b}-c_{a}}{S_{\mathrm{SLs}}}$

Where $c_{b}$ is the quality before separation in $\mathrm{g}, c_{a}$ is the quality after separation in $\mathrm{g}, S_{\mathrm{SLs}}$ is the isolated SLs in $\mathrm{g}$.

bSLs separation rate $=\frac{S_{\mathrm{SLs}}}{c_{\mathrm{SLs}}}$

Where $S_{\mathrm{SLs}}$ is the isolated SLs in $\mathrm{g}, c_{\mathrm{SLs}}$ is the quality of SLs before separation in g.

$\mathrm{cIn}-$ situ separation efficiency $=\frac{S_{\mathrm{SLs}}}{t}$

Where $S_{\text {SLs }}$ is the isolated SLs in $g, t$ is separation of time in min.

Table 2 Comparison between fed-batch fermentation and semi-continuous fermentation

\begin{tabular}{llllll}
\hline Strain & substrate & Fermentation period $(\mathrm{h})$ & Productivity $(\mathrm{g} / \mathrm{L} / \mathrm{h})$ & Yield $(\mathrm{g} / \mathrm{g})$ & $\mathrm{Q}_{\mathrm{P} / \mathrm{DCW}}(\mathrm{g} /$ \\
\hline C. bombicola & Glucose, rapeseed oil & 168 & 1.70 & 0.47 & 0.077 \\
C. bombicola & Glucose, rapeseed oil & 378 & 2.15 & 0.58 & 0.096 \\
C. bombicola & Glucose, rapeseed oil & 221 & 1.55 & 0.48 & 0.068 \\
C. ${\text { albicans } \text { O-13-1 }^{\mathrm{b}}}^{\mathrm{b}}$ & Glucose, oleic acid & 300 & 1.59 & 0.60 & 0.023 \\
C. bombicola & Glucose, rapeseed oil & 1023 & 0.61 & 0.53 & 0.019 \\
C. bombicola & Glucose, waste food & 480 & 2.39 & 0.73 & 0.020 \\
\hline
\end{tabular}

a Fed-batch fermentation 
b Semi-continuous fermentation 